\title{
Analysis of the Teaching Art of Leaving Blank in Chinese Classroom in Middle School
}

\author{
Xie Lu, Xiao Jiugen* \\ Research Center of Language and Language Life, Jiangxi Normal University, Nanchang, China \\ Email address: \\ 492933734@qq.com (Xie Lu),jxsdxjg666666@sina.com (Xiao Jiugen) \\ ${ }^{*}$ Corresponding author
}

\section{To cite this article:}

Xie Lu, Xiao Jiugen. Analysis of the Teaching Art of Leaving Blank in Chinese Classroom in Middle School. Science Innovation. Vol. 8, No. 1, 2020, pp. 1-4. doi: 10.11648/j.si.20200801.13

Received: December 26, 2019; Accepted: January 21, 2020; Published: February 4, 2020

\begin{abstract}
The art of leaving blank originated from the technique of expression in Chinese painting. It has been widely used in literary works, and also creatively used in classroom teaching, such as novels, poems, essays, etc. In terms of fiction, the teaching art of leaving blank in classroom has a positive significance for optimizing classroom structure and improving teaching effectiveness. The use of the teaching art of leaving blank in classroom not only enhances the ability of the teacher to interpret the work, masters the structure and content of the work skillfully, but also improve the students' appreciation level and aesthetic taste, and most importantly, it can stimulate the students' potential creativity. The teaching art of leaving blank in novel teaching has its profound theoretical foundation and a large number of practical cases. It can not only construct vivid characters, taste rich ideological connotations, comprehend profound thematic significance, but also cultivate students' thinking ability, improve their intelligence level and then create a language class which is efficient and vibrant.
\end{abstract}

Keywords: Chinese Teaching, Novel Analysis, The Teaching Art of Leaving Blank, Optimize the Structure, Improve Effect

\section{中学语文教学中小说留白艺术分析}

谢璐, 肖九根

江西师范大学语言与语言生活中心, 南昌, 中国

邮箱

492933734@qq.com（谢璐）, jxsdxjg666666@sina.com（肖九根）

摘要: 留白艺术起源于中国绘画虚实相生的表达技法, 但它在文学作品中也得了广泛的运用,而且还创造性地运用于基 础语文诸如小说、诗歌、散文等多种文体的课堂教学之中。以小说而言, 课堂教学运用留白艺术, 对于优化课堂结构, 提高教学效果有着积极的意义。课堂教学运用留白艺术, 不仅教师能增强作品的解读能力, 涃熟地驾驭作品的结构内 容,还能提高学生的欣赏水平和审美情趣, 而最为重要的是能激发学生潜在的创造力。小说教学运用留白艺术有其深厚的 理论基础, 也有其大量的实践案例, 它不但可以建构生动的人物形象, 品尝丰富的思想内涵, 领悟深刻的主题意义, 还能培养学生的思维能力, 提高他们的智能水平, 进而打造出一堂既富高效又充满生机与活力的语文课。

关键词：语文教学, 小说分析, 留白艺术, 优化结构, 提高效果 


\section{1. 引言}

留白艺术源于中国传统绘画计白当黑的方法, 以达到 虚实相生的艺术表达效果, 给人留下无穷的韵味。留白的 美学概念不仅在绘画等艺术中广泛运用, 而且在文学作品 中也有诸多体现。以语文教材中的小说为例, 就有人物留 白、情节留白、语言留白等。留白艺术是小说常用的一种 技巧, 小说借助留白这一虚实相生的表达艺术, 使之更具 可读性和生命力。

留白艺术运用于语文课堂中的小说教学, 无论对于教 师还是学生均有一定的教学意义和实践价值。首先, 教学 中运用留白艺术, 这就要求教师对小说文本要有深刻的理 解, 对课堂教学内容要有驾驭能力, 从而使之得到锻炼而 成长起来。其次, 教学中运用留白艺术, 有利于学生的智 能转化, 使之发展思维能力。这是因为留白艺术的运用, 有助于在思考中充分地运用自己的知识和经验, 这就是学 生知识与能力的转化过程, 也是思维的发展过程。[1] (第 10页) 再次, 教学中运用留白艺术, 还有利于提高学生的 审美情趣和欣赏能力。不论叙事性还是抒情性小说, 都是 语文教学中的重要内容。运用留白艺术进行教学, 对于培 养学生的审美情趣, 提高他们的品赏领悟能力, 都有十分 积极的意义。最后, 教学中运用留白艺术, 更为重要的是 有助于学生以自身的审美经验去填补作品, 把课堂教学变 成美的艺术, 从而激发学生的再创作。

在现行的小说教学中, “情节一人物一环境”的教学模 式以及教师、教参的解读充斥着课堂, 不仅将各具特色的 作品等同对待, 而且还忽视学生的阅读体验和真实情感。 《义务教育语文课程标准（2011年版）》明确指出：“正 确把握语文教育的特点, 积极倡导自主、合作、探究的学 习方式，努力建设开放而有活力的语文课堂。”[2]（第2 页) 新的语文课标要求课堂教学不是“一言堂”, 而要注重 学生语文能力的发展。语文教学应坚持“学生语用发展本 位”，并通过重建积极的语用来释放学习者心智活力的“表 达本位” 新范式。[3] (第20页) 如何把小说经典篇目教出 韵味, 如何培养出有较高语文素养的学生, 这值得每位语 文教师深思。留白艺术与小说教学相结合, 为这个问题提 供了一种新思路、新方法。留白艺术进入语文课堂教学, 不仅是当前教育改革的需要, 而且也有其理论基础和实际 应用价值。

\section{2. 留白艺术应用于小说教学的理论基础}

从中国美学思想发展史看, 留白艺术可以上溯到春秋 战国时期的道家思想。如《老子》第四十一章云：“大音 希声，大象无形。”大音”不可闻，“大象”不可见，于不可 闻和不可见之中衍生出无限的可能性, 而可能性本身又不 能脱离有形有名的实物而独立存在, 二者是相辅相生的。 中国艺术作品强调意义的空白便是受此影响而发展起来 的, 如小说中不可言明的情感就是如此。

从建构主义理论看, 其强调学生应主动建构自己的知 识结构体系, 认为学生学的知识被赋予了心理意义的“主 观信息”。[4] (第11页) 学生应把新知识与旧信息进行加 工整合, 使之融为一体, 从而扩展原有的认知。因此, 教
师进行小说教学时, 应适当地留下解读的空白, 把补白的 机会留给学生, 这对提高他们的智能水平, 发展他们的思 维能力大有禆益。

从接受美学理论看, 留白艺术运用于语文教学有其存 在的基础。在接受美学视域里, 文本的意义是作者与读者 一起完成的。姚斯认为, 一个作品, 即使是印成书, 读者 阅读之前, 也只是半完成品。[5] (第70页) 只有在读者的 阅读和解释条件之下, 文本才成为作品。小说也是如此, 教师和学生都是它的读者, 由于自身审美经验的不同, 他 们对作品的理解也有所不同。但是, 长期以来, 教师总是 主宰着小说的文本解读, 学生亦步亦趋, 没有起到主体应 有的作用。语文课堂教学是一门艺术, 其教学过程是一个 双方互动的多元性过程, 而非单元性的。[6]（第178页） 因此, 教师有必要运用留白教学艺术, 以彰显学生独自解 读作品的能力。

\section{3. 小说教学中留白艺术的运用方法}

小说不同于诗歌、散文等体裁, 它源于生活又高于生活, 通过语言艺术“细致的人物刻画、完整的故事情节、背景或 环境的具体描绘”来反映生活。[7]（第172-176页）进行小说 教学, 必须构建小说独特的教学方式。小说教学中运用留白 艺术，能够帮助学生“克服现有的语文经验与课文理解、感 受所需要的语文经验之间的落差”，[8]（第23-26页）从而更 好地理解小说文本, 读出自己心中的味道。

\section{1. 以讲解留白艺术建构作品生动的人物形象}

众所周知, 人物是小说的灵魂。一篇优秀的小说, 必 定是枢密有致, 有让读者想象的余地以及印象深刻的人物 形象。实际上, 人物形象本身也是有留白的, 需要学生根 据自身经验对人物形象进行再加工。[9] (第102页) 例如, 中学语文课文《故乡》就多处留有空白。作者描写少年闰 土时回忆了诸多往事: 捕鸟雀、月下守瓜田、与闰土分别, 等等。而在描写中年闰土时, 作者只用了胗骞数语描写闰 土的外貌、语言。显然, 闰土的心境已经发生了很大变化。 在这样的对比之下, 淳朴、天真的少年与麻木、寻常的农 民形象跃然而上。教师教学时就不能忽略作者描写中年闰 土形象时有意而为的留白艺术。

教学时, 面对作品的留白, 我们需要对之补白。补白 是补充交待人物的心理、情节的发展变化。[10]（第22页） 补白不是要教师唱独角戏, 学生也应进入作品角色, 发挥 想象, 补充人物形象。例如, 讲授《故乡》, 教师运用留 白艺术, 借助表格, 让学生对人物心理变化及其原因等进 行补白。首先, 教师出示PPT, 展示下表:

表1 《故乡》教学设计表。

\begin{tabular}{lll}
\hline & 少年闰土 & 中年闰土 \\
\hline 外貌 & \\
语言 & \\
动作神态 & \\
对我的态度 & \\
对生活的态度 & \\
变化原因 (表层和深层) & \\
\hline
\end{tabular}


此表将少年闰土与中年闰土囊括在内, 教师可先做点 示范。例如, 少年闰土毫无拘束地与“我”交谈, 中年闰土 恭敬地叫“我”老爷......剩下的空白则由学生填补。然后, 以表上内容为依据, 让学生再次回顾课文内容, 这样不仅 有利于提高学生的分析能力, 更为重要的是有利于培养他 们的智能思维能力。

人们不难发现，小说中作者仅是将他看到的闰土进行 描写, 而对其所发生变化的原因只字未提。此时, 教师就 应展示作品的写作背景来帮助学生理解, 启发他们结合社 会时代背景来挖掘它的深层原因, 得出作者塑造的并不是 一个被生活压弯了腰的闰土, 而是千千万万像闰土这样被 社会压迫向命运低头的下层劳动者形象。

再如中学语文课文《范进中举》, 篇幅较长, 若要讲 全、讲细, 在当今的教学任务要求下是不可能的。因此, 教师可以打破传统的从上至下授课方式, 不再串讲, 而是 采用留白艺术方法, 隐去教师的角色, 省去繁琐的讲解, 让学生担当课堂主角, 完成教学内容。例如, 以“寻找范 进变疯的原因”为线索, 去分析范进的人物形象。以三、 四个同学为一组去阅读作品, 寻找其中原因。之后, 每组 派个代表来宣讲本组所分析的原因, 其他同学在听的过程 中可以进行补充。如此一来, 范进中举发疯的原因就易分 析了。范进之前屡试不第, 年逾五十中个秀才, 这已是一 件高兴的事了; 不料, 是年运星高照, 他不仅中了秀才, 还中了举人。如此一来, 他大喜若狂, 终而乐极生悲, “痰 涌上来, 迷了心窍”。此时教师可趁热打铁, 再次追问: “范 进变疯仅是他自身的原因吗? 如果不是其深层原因又是 什么呢? ”由此触及深层内核——封建制度的弊端, 从而 揭示封建社会选拔人才的㽽疾是造成千千万万个范进中 举发疯的根本原因。

通过《范进中举》的人物分析, 学生在找寻、思考中 获得答案, 这就是留白所发挥的作用。此时, 教师“无声” 胜有声, 因为课堂以学生为主体, 已经充分调动了他们的 积极性和主动性, 锻炼了他们的思维能力, 这有利于提高 他们的创新本领, 从而使其在思想碰撞的火花中再次建构 人物形象, 完善知识体系。

\section{2. 以品后留白艺术留下作品丰富的内容韵味}

一堂课以何种方式教学才能回味无穷呢? 如要达到 这一效果, 非得以品后留白艺术不可, 因为这一艺术能给 学生以思考的时间, 给学生以想象的空间, 给学生以咀嚼 的甜味, 使之实现最佳的教学效果, 也能使学生的知识结 构达到最优化的组合。尽管品后留白艺术具有多种方式, 但无论哪种方式, 都能收到意想不到的效果。

譬如, 设置一个悬念, 不仅能激发学生强烈的求知欲, 还能培养学生的发散性思维, 将其思维从课内引向更加广 阔的天地。《孔乙己》一文的结尾是这样说的: “大约孔 乙己的确是死了。”教师在这可进行巧妙的假设：“大约” 而不是“的确”，[11]（第77页）假如孔乙己没死呢? 假如 他一改之前迂腐性格与命运抗争呢? 假如......这样的“假 如”还可以有不少。教学《装在套子里的人》一文, 如问: “与世隔绝的别里科夫要恋爱了, 他从套子里探出头来。 同学们不妨想一想, 这样的人会得到姑娘的爱吗? 为什
么? 结局又会怎样? ”这样以问题设置悬念, 既引导学生 去阅读课文, 又让他们展开自由的翅膀去想象......这种品 后留白艺术, 能够调动学生学习的积极性, 增强他们的求 知欲, 促使他们去深读课文。

至于抒情性很强的作品, 如《孤独之旅》这篇诗化小 说, 不单语言美, 还富有含蓄美、意境美, 采用默读细品 领悟的留白艺术能起到意想不到的效果。如在静默细品的 过程中, 杜小康的孤独弥漫着学生的心里, 此时倘若配上 点音乐, 想必更能深悟作品主人公细淢的心理变化。此时, 教师要求学生用“内在的眼”去看作者描绘的世界, 用 “内 在的耳”去倾听作者的心声, 就别有一洞天地了。[12]（第 53页) 这种“沉浸式”阅读, 最有利于增强学生的审美体验。

一堂课的结束不是学习的终点, 而是拓展的起点。文 学作品, 其语言艺术、思想情感、结构布局等, 这些审美 成分有时是一种潜在的、隐藏的表现, 需要我们对此进行 梳理、探究和整合。[13] (第154页) 教师可以充分运用品 后留白艺术去拓展学生的知识领域, 去体会潜藏的审美成 分。不论设置悬念还是静默细品, 其目的都是构建高效语 文教学课堂, 唤起学生的主体意识, 使其不断地去进行探 索。

\section{3. 以问题留白艺术探究作品深刻的主题意义}

语文教学过程中, 提问是教师用得最多的方法。好的 问题不仅对学生有启迪意义, 而且对于提高语文教学效果 也起着至关重要的作用。怎样的问题才是好问题呢? 怎样 的提问才能发挥最大的效能呢? 运用问题留白艺术, 能给 予很好的解决。所谓问题留白艺术, 涉及教师与学生提问 两方面。以教师而言, 钱梦龙先生所说的“问宜曲”就是一 种问题留白艺术。[14]（第21-23页）“曲问”是相对于“直 问”而存在的, 它是变换角度提问的一种艺术, 让学生进 行多维思考。就学生来说, 它是对问题提出质疑的一种方 式。教师把“提问权”交给学生, 是鼓励他们对问题的自我 质疑。[13]（第53页）因此, 从某种意义上说, 教师的教 不是为了消除疑问, 而是启发学生对问题进行思考, 培养 他们勤于思考的习惯, 从而提高他们的思维能力, 这也是 教学效果的真实显现。

钱梦龙先生在教《多收了三五斗》时, 以这样的问题 启发学生理解作品的主旨。他问: “万盛米行的先生对农 民说话是有气无力、鄙夷不屑的, 而万源祥等几家商店的 伙计却不惜工本地叫着“乡亲”, 同是在一条街上做生意的, 为什么态度这样不同? ”钱先生目的在于讲解小说的主 旨: 米行老板借粮食丰收垄断市场, 剥削农民, 以致造成 了农村市场萧条、农民日趋贫困破产的社会现实。不过, 为了揭示这个主旨, 钱先生采用了“曲问”的教学方法, 即 在米行老板态度前后矛盾之处设问, 这比直接问学生“小 说的主旨是什么”更巧妙, 更有艺术性。乍一听, 似乎在 问米行老板借机逐利, 其实并非如此。学生必须深思, 方 能透过现象看本质, 这就强化了学生的思辨能力。

问题留白艺术, 实际上就是一个生疑与释疑的过程。 学生是语文学习的主体, 教师有效的等待时间就是让学生 冲动的本能直觉转变为理性的深入思考, 让提问成为师生 之间的双边互动。[15] (第14页) 有的作品主旨难以环磨, 
即使通读通讲全篇, 学生依旧疑惑不解, 如果以问题的形 式提出, 往往峰回路转, 柳暗花明。如《最后一课》, 对 于初一学生来说, 还难以体悟韩麦尔先生沉重而不舍的情 感, 因为其事件离他们太远。这就有必要采用问题留白的 方法进行教学, 以学生提问为主体, 主动权在学生一方, 教师仅是对问题起个“导”的作用, 其职责是全程监控学生 懂与不懂的动态而已。

有言道: 授人以鱼不如授人以渔，教师教的应该是方 法, 而不是过程。以问题为导向的留白艺术, 不仅能让学 生学会分析问题, 更为重要的是锻炼学生的思维能力, 从 而提高他们的智能水平。

\section{4. 结语}

总而言之, 留白艺术运用于语文小说教学中有诸多意 义。于教师而言, 它是知识功力的一种检视, 也是教学经 验的一种演示; 于学生而言, 它是参与课堂活动的动员令, 也是进行思维活动的催化剂; 于作品而言, 它是意义内涵 的一种宣言, 也是自身生命的一种延续。在语文教学中, 运用留白艺术来建构人物形象, 品味思想内容, 探究主题 意义, 这是提高语文教学质量有效途径之一。诚然, 留白 艺术的运用不能模式化, 它强调留白中孕育着希望的光 芒, 在适时适度留白艺术的运用中打造出一堂既富高效又 充满生机与活力的语文课。

\section{参考文献}

[1] Wang Xiaoyu. The teaching art of leaving blank in Chinese classroom construction in high school [D]. Central China Normal University, 2018.
[2] 教育部.九年义务教育语文课程标准[S].北京:中华人民共和 国教育部，2011.

[3] 潘涌.积极语用, 为真语文教学注入科学内涵[J]. 语文建设, 2015(08).

[4] 莲蓉, 罗丽芳.教育心理学概论[M].北京: 北京师范大学出 版社, 2009.

[5] 朱立元. 接受美学导论[M].合肥: 安徽教育出版社, 2004 .

[6] Hong Biqun. On the teaching art of leaving blank in Chinese classroom teaching [J]. Consume Guide, 2008 (12).

[7] 王荣生, 宋冬生.语文学科知识与教学能力 [M].北京:高等教 育出版社, 2011.

[8] 荣维东. 语文文本解读实用教程[M]. 北京: 北京大学出版社, 2016.

[9] 万鲁丹.运用留白艺术进行语文教学的探究 [J].汉字文化, 2018(10).

[10] 覃佐菊.留白与补白一阅读教学中人物内心活动的两种 处理方式[J].中学语文教学参考, 2017(23).

[11] Chen Yunlong. Glimpse the teaching art of leaving blank in junior high school Chinese classroom teaching [J]. Modern Chinese, 2016 (11).

[12] 计敏芳.最美,是那一瞬的留白一一例析初中语文课堂教学 中的"留白"艺术[J].中学语文教学参考, 2015 (11).

[13] 范依琳.刍议中学语文课堂教学中的留白艺术 $[\mathrm{J}]$.现代基础 教育研究, 2014 (13).

[14] Cao Ling. The exploration of Qian Menglong's questioning art [D]. Central China Normal University, 2018.

[15] 沈艳芬, 无画亦成妙境, 留白点石成金[J].中学语文教学参 考，2018(08). 